\title{
LA DOUANE A LA FRONTIERE DE LA REPUBLIQUE DEMOCRATIQUE DU CONGO ET LA ZAMBIE : ORGANISATION JURIDIQUE ET CONDITIONS GENE RALES
}

\author{
Par Musonda Bwalya E. Didier et Nkiko Mutabazi Vivien ${ }^{1}$
}

\section{INTRODUCTION}

Parler de la douane à la frontière de la République Démocratique du Congo avec la Zambie, revient à résumer, à définir, à commenter les différentes activités administratives qui se déroulent à l'occasion des opérations d'exportation et d' importation afin d'encourager les différentes améliorations et de relever les imperfections de manière à définir une ligne de conduite sous forme de lege ferenda afin que la douane soit une véritable source des recettes pour la république toute entière en dépit de son caractère déjà meilleur qu'il Ya 20 ans, malgré la multiplicité des services sans base juridique œuvrant à cette frontière.

L'étude que nous menons se réfère au processus de réforme politique et économique ayant pris cours en République du Zaïre depuis le 24 avril $1990^{2}$, nous constaterons qu'il n'a pas encore permis de restaurer complètement les conditions qui permettraient de bâtir dans ce pays une troisième république qui serait régie par les principes de droit et de justice dans tous les domaines en général et dans les domaines générateurs des recettes en particulier.

En effet, parlant de la douane, une situation est observée selon un agent de la direction générale de douane et accises (DGDA en sigle), une inquiétude jonche l'organisation de la douane à la frontière avec la Zambie étant donné la multiplicité des services qui œuvrent à la douane sans autorisation et ceci sous l'œil impuissant des autorités. Ce comportement laisse planer des doutes et incertitudes de l'agent travaillant à la douane au point de se laisser tremper dans une incertitude qui remet en cause son efficacité au travail et se lance dans une histoire de fraude mettant en péril la crédibilité de la douane.

Ainsi, dans le présent travail, il ne sera pas question pour nous de rechercher les causes académiques de la crise politico-économique en République Démocratique du Congo ni de rechercher les coupables de quelques crimes que ce soit, mais son seul but et objectif est de constater, de commenter, la situation générale qui prévaut dans les postes frontaliers en République démocratique du Congo et en particulier ceux qui se retrouvent à la frontière avec la Zambie notamment : Kasumbalesa, Tshinsenda, Mokambo, Pweto. etc. afin d'en tirer des conséquences tant sur le plan social que de droit en vue de proposer une méthodologie des

1 Tous Assistants à l'Université de Lubumbashi, Faculté de Droit.

2 Cette date marque le discours du Président MOBUTU consacrant le multipartisme politique. 
solutions pratiques qui pourraient concourir à mettre fin à la persistance de l'anarchie, des désordres et des tracasseries dus, outre les raisons ci-dessus évoquées, par la multiplicité des services de l'Etat aux postes frontaliers ci-haut évoqués et ce nonobstant les textes légaux et réglementaires pris à cet égard réduisant à 4 services devant œuvrer à chaque poste frontalier de la république démocratique du Congo en vue d'assainir réellement les postes frontaliers en république démocratique du Congo.

Et pour y arriver la présente réflexion est organisée, outre cette introduction en deux points dans lesquels nous présenterons au premier point l'organisation juridique de la douane en république démocratique du Congo, au second point, les conditions juridiques de la douane sur toute l'étendue du territoire en général et à la douane partageant la frontière avec la Zambie en particulier et enfin en guise de conclusion cette étude se proposera une liste des perspectives sous forme de lege ferenda.

Cela étant, quelle est alors l'organisation juridique de la douane en république démocratique du Congo à travers les textes légaux et réglementaires en vigueur et qui s'applique, mutatis-mutandis, à la douane de Kasumbalesa?

\section{ORGANISATION JURIDIQUE DE LA DOUANE EN REPUBLIQUE DEMOCRATIQUE DU CONGO}

Ce point se préoccupera d'examiner le contenu des textes légaux et réglementaires ayant régi la douane en république Démocratique du Congo depuis 1885 jusqu'aux récentes réformes douanières de 2010 mais avant d'y arriver nous tenterons de faire une petite notion sur la douane.

\section{Généralités}

La douane: droits et moyens d'action.

La douane et les droits de douane ne sont pas une création récente des Etats. Claude J. BERR et Henri TREMEAU soutiennent que la prescription de droits de douane et taxes lors $\mathrm{du}$ franchissement d'une frontière par une marchandise remonte à l'antiquité. Elle n'avait alors eu pendant des siècles qu'une justification fiscale.

Dans une organisation financière encore primitive, taxe de circulation et droits de passage constituaient les moyens les plus simples de lever l'impôt. Jusqu'à la renaissance, les taxes douanières ne furent d'ailleurs prélevées, mieux valait frapper les produits destinés à l'étranger plutôt que les produits étrangers achetés par les nationaux.

A cette préoccupation s'ajoutait le souci d'assurer le ravitaillement du pays et d'éviter les famines. Il faudra, en fait, attendre le $17^{\text {ieme }}$ siècle pour que se fasse jour l'idée selon laquelle le droit de douane pouvait jouer un rôle économique en favorisant la naissance et le développement de certaines industries manufacturières. ${ }^{3}$

3 BERR C.J., TREMEAU H., le droit douanier, $5^{\text {ieme }}$ ed. Economia, paris, 2001, p2. 
A Rome, au temps des rois, écrit J.R ESSO ABONGO MOBUKA, on utilisait déjà les « portoria » ou droits perçus dans les ports, à l'entrée et à la sortie des marchandises.

Par ailleurs, on reconnaît souvent à la confiscation des marchandises de fraude. Quand la douane, étendit ses possessions en orient, Rome désigna, pour recouvrer les taxes de douane et de péages, des receveurs dont le plus célèbre fut Mathieu.

Le futur apôtre du christ cumulait les fonctions de douane et de percepteur a capharnaüm, lieu de passage des cadavres venant de l'orient et l'importante ville frontière de Galilée sur les bords du lacs de Tibériade, suite à la guerre provoquée par le droit de péage en Gaule, César fixa l'impôt douanier à $1 / 4$ de la valeur des marchandises, fait qui marque l'apparition des droits ad-valorem. D'où le droit de douane devint l'impôt.

Ces droits étaient perçus à l'époque des mérovingiens et des carolingiens. On les appelait « tenlieum » ou « tonlieux ». Ils étaient perçus à l'occasion soit d'entrée, soit de sortie soit encore de la circulation des marchandises. ${ }^{4}$

L'évolution de la douane n'a pas manqué d'affecter la désignation même du service. Jean-Luc Albert écrit à ce propos que le mot « douane »a été souvent analysé et commenté. Certains lui trouvent une origine arabe (au $7^{\text {ieme }}$ siècle) au travers du terme « dawana $»^{5}$ ou « diwana ».

Il aurait connu des itinéraires divers : italien (doane), espagnol, français enfin avec les termes (dohane) 1291, (doaner) et (doan), 2441. Le terme (douaner) remontrait en France en 1675 et caractériserait une opération de marquage de marchandises avec le « plomb de l'administration $»{ }^{6}$

Ainsi entendu, le mot douane peut désigner à la fois l'institution d'un impôt frappant les marchandises importées ou exportées, les services qui l'ont en charge, les bâtiments qui les abritent et les lieux où ceux-ci sont établis. Ainsi, nous avons d'une part les droits de douane désignant les impôts particuliers sur la dépense, perçus à l'occasion de l'importation ou de l'exportation des marchandises et d'autres part l'administration des douanes désignant l'ensemble des services chargés de l'assiette, de la liquidation et de perception des droits de douane ainsi que du contrôle des marchandises lors du franchissement d'une frontière. $^{7}$

2. Fondement juridique de la douane en République Démocratique du Congo.

Le fondement juridique de la douane en République Démocratique du Congo est à rechercher dans l'histoire connue du commerce de notre pays avec des Etats ou ressortissants d'Etats étrangers, sans toutefois prétendre à la même ancienneté.

4 ESSO ABONGO, JR., La douane et les droits de douane: office des douanes et accises à l'épreuve de l'exégèse administrative. Presse de l'institut technique Salama, 2009, p.97.

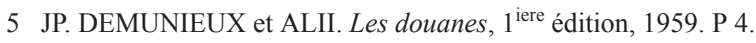

6 ALBERT, J., douane et droit douanier: questions judiciaires. PUF, Paris, 2013, p. 23.

7 GERARD CORNU, vocabulaire juridique, $3^{\text {ieme }}$ edition, juillet 1992. 
David van REYBROUCK écrit à ce sujet que sous son règne qui dura quatre décennies (1506-1543) NZINGA MVEMBA dit Alphonse $1^{\mathrm{er}}$, roi des kongo, appui sa puissance sur le commerce avec les portugais. ${ }^{8}$ Un extrait d'une lettre écrite des mains du roi Alphonse $1^{\text {er }}$ au roi jean III du Portugal en 1526 confirme l'affirmation ci-dessous : « beaucoup de nos sujets convoitent vivement les marchandises du Portugal, que les vôtres apportent en nos royaumes ${ }^{9}$ dans la suite de l'histoire de la douane en République Démocratique du Congo précisément avec l'Acte Général de Berlin du 26 février 1885.

Notons qu'il est le premier texte ayant consacré la liberté de commerce sur le bassin du Congo qui se résume en ces termes : «le commerce de toutes les nations jouira d'une complète liberté $\left(\operatorname{art.~} 1^{\mathrm{er}}\right)$. Les marchandises de toute provenance importées dans ces territoires, sous quelque pavillon que ce soit, par la voie maritime, fluviale ou par celle de terre, n'auront à acquitter d'autres taxes que celle qui pourraient être perçues comme équitable compensation de dépenses utiles pour le commerce et qui, à ce titre, devront être également supportés par les nationaux et par les étrangers de toute nationalité. Tout traitement différentiel est interdit à l'égard des navires comme des marchandises(art. 3) les marchandises importées dans ces territoires resteront affranchies de droits d'entrée et de transit. Les puissances se réservent le droit de décider, au terme d'une période de vingt années, si la franchise d'entrée sera ou non maintenue (art. 5). »

Il est donc constaté qu'en République Démocratique du Congo, la douane est congénitale à l'Etat et ce, depuis l'Etat indépendant du Congo, par l'interdiction de perception ou la franchise de droits d'entrée.

Vers la fin de 1885, le roi Léopold II ordonna par décret la perception des droits de sortie. ${ }^{10}$

Dès 1886, les textes juridiques se sont multipliés pour une meilleur administration de la colonie, et par là même, de la douane. Ainsi, le 25 mars 1886, un arrêté portait modalités de perception des droits de sortie tandis qu'un autre arrêté instituait un règlement général des douanes.

L'annexion le 15 novembre 1908 par la Belgique de l'ancienne propriété du roi marquera l'histoire par la structuration qu'elle apporta à l'administration au Congo. A Anvers, l'office douanier colonial sera institué par la loi du 20 novembre 1919. Elle avait compétence pour recevoir les déclarations d'importation de marchandises à destination ou en provenance du Congo. C'est donc à Anvers que s'effectuaient les opérations de dédouanement pour le compte de la colonie.

Deux autres textes datant de l'époque coloniale constitueront plus tard la législation douanière du Congo et continueront à régir les opérations douanières pendant un peu plus de soixante ans. Il s'agit du décret du 29 janvier 1949 coordonnant et révisant le régime

8 REYBROUCK D, V., Congo, une histoire, ACTES SUD, Amsterdam, 2012, p.28.

9 Citée par HOCHSCHILD A., les fantômes du roi Léopold: un holocauste oublié, Belfond, paris, 1998, p.23.

10 Décret du roi souverain du 15 décembre 1885. 
douanier et de l'arrêté $n^{\circ}$ 33/9 du 6 janvier 1950 portant règlement d'exécution du décretdu 29 janvier 1949.

Après l'indépendance, ces textes connurent maintes modifications pour les adapter aux nécessités du temps. C'est le 20 février 2011, avec l'entrée en vigueur du code des douanes porté par l'ordonnance-loi $n^{\circ} 10-002$ du 20 aout 2010, que ces textes cessèrent d'exister.

Au sens du code des douanes ainsi institué, l'administration des douanes ou douane est l'administration ou l'organisme public chargé (e) de l'application de la législation douanière et de la perception des douanes et taxes à l'importation et à l'exportation. Il est également chargé (e) de l'application d'autres lois et règlements relatifs à l'importation et à l'exportation.

L'administration congolaise des douanes est, depuis le 3 décembre $2009^{11}$, un service public de l'Etat dénommé « direction générale des douanes et accises, en sigle DGDA. Le directeur général des douanes et accises en est l'autorité qui exerce les plus hautes fonctions dans la gestion courante ${ }^{12}$. Il est dans sa charge assisté de deux directeurs généraux adjoints.

L'hygiène et la salubrité publique ne sont pas en reste dans l'organisation de la douane en République Démocratique du Congo, ces matières étaient régies par le décret du 19 juillet 1926.

L'ordonnance $n^{\circ} 83-033$ du 12 septembre 1983 quant à elle réglementait la police des étrangers en République Démocratique du Congo.

\section{Organisation administrative :}

Conformément au décret $\mathrm{n}^{\circ}$ 011/06 du 25 janvier portant cadre organique de la Direction Générale des douanes et accises, la DGDA comprend :

- Une administration centrale ayant des directions et des services centraux ainsi qu'une brigade des douanes (Bureau de Coordination, Direction des Ressources Humaines, direction de réglementation et facilitation, direction de la brigade et lutte contre la fraude, Direction du Tarif règle d'origine, Direction de la valeur, Direction des huiles minérales, Direction des autres produits d'accises, Direction des Recettes du Trésor, Direction des Finances internes, Direction des Equipements et Logistiques, Direction des Statistiques, Documentations et Etudes Economique, Direction des affaires Juridiques et Contentieux, Directions des systèmes et technologies d'information, Direction de l'audit interne, Direction de reformes et modernisation;

- Des directions provinciales (ville de Kinshasa, Kinshasa aéroport, Bas Congo, Equateur, province orientale, nord Kivu, Kasaï Orientale, Kasaï Occidental, Sud Kivu, Katanga, Bandundu, Maniema) et inspections provinciales ainsi que des bureaux de douane;

11 Cfr. Décret $n^{\circ}$ 09/43 de 3 décembre 2009 portant création de la direction générale des douanes et accises.

$12 \mathrm{Cfr}$ art. 5 du code des douanes. 
- Des représentations de la douane à l'étranger (représentation auprès de l'organisation mondiale des douanes, représentation à Mombasa et, depuis peu, à Dar es Salam).

En fait, la direction générale fonctionne en services centraux et provinciaux. Elle est l'organe général qui s'occupe essentiellement de l'administration et n'entre pas dans les opérations alors que les directions provinciales sont chargées de la gestion des opérations de dédouanements et autres. Et le cadre juridique de ces opérations est principalement constitué par le code des douanes institué par l'ordonnance-loi $n^{\circ} 10-002$ du 20 août 2010 et ses mesures d'application ainsi que le tarif des droits et taxes à l'importation.

La direction provinciale est dirigée quant à elle par un directeur provincial et cette direction s'éclate à son tour en services provinciaux sous la direction de sous directeurs; notons que la direction provinciale peut créer des bureaux de douane sous la direction d'un sous-directeur ou d'un inspecteur provincial. C'est le cas de la douane à Kasumbalesa qui a deux bureaux de douane dont l'un dépend de l'autre.

Il s'agit du bureau frontalier dont le code est 704/B et celui de Whisky, bureau intérieur dont le code est 722/B.

Après tout ce qui précède, quelles sont les conditions générales de toute douane en République Démocratique du Congo en général et celle qui partage la frontière avec la Zambie en particulier?

4. Conflits et compétences dans l'administration douanière à la frontière avec la Zambie.

Conformément à l'article $1^{\text {er }}$ du décret précité du 08 mars 2008 qui dispose « sont seuls habilités à exercer aux frontières, dans le circuit de circulation des personnes et/ou des marchandises les services et organismes ci-après :

1. L'office de douane et accises, en abrégé " DGDA » pour les formalités de dédouanement des marchandises, à l'importation et à l'exportation. certes, dépuis le Code des douanes et le décret $n^{\circ} 09 / 43$ du 3 décembre 2009 portant création et organisation de la Direction générale des douanes et accises (DGDA), il a été assigné à l'administration douanière congolaise des missions de plus en plus importantes avec des prérogatives de puissance publique, dont l'accomplissement avait nécessité une couverture juridique pour ses Cadres et Agents. Il a été décidé ainsi dans le but de renforcer le dispositif de lutte contre la fraude étant donné que dans la perspective de la refondation de l'Etat, la république Démocratique du Congo a besoin des fonds. Aussi, la mobilisation des recettes publiques et la rationalité dans leurs dépenses nécessitent-elles des mesures appropriées pour freiner l'hémorragie financière qui constitue un manque à gagner criant pour le Trésor. Ainsi, depuis le vendredi 10 février 2012, la direction des douanes et accises s'est enrichie de 71 nouveaux Officiers de Police Judiciaire, en complément aux 193 officiers de police judiciaire (OPJ) lancés sur le terrain depuis décembre 2011. Ce corps a pour principale mission : traquer les fossoyeurs de la douane.

2. l'office congolais de contrôle en sigle (OCC). 
En sa qualité d'organisme public à caractère technique et scientifique, elle a pour missions légales :

- Préserver les intérêts économiques en assurant les contrôles de la qualité, de la quantité et de la conformité de toutes les marchandises;

- Analyser tous les échantillons et produits pour protéger les clients;

- Garantir la sécurité des installations, machines, appareils, travaux et étalonnages;

- Prévenir les sinistres et procéder au constat des dommages ou des avaries;

- Exercer toutes opérations quelconques se rapportant directement à son activité légale, sauf les opérations de l'achat en vue de la revente. ${ }^{13}$

- A la frontière, elle est utile pour contrôler au lieu d'embarquement, de la qualité, de quantité et des prix des marchandises et produits exportés et importés.

3. La direction générale d'immigration en sigle (DGM). Ce service de l'Etat s'occupe des mouvements des personnes physiques, à l'arrivée et au départ.

4. Les services d'hygiène publique pour les contrôles sanitaires.

Notons que partout où il Ya une frontière en république Démocratique du Congo, il doit y avoir ces 4 services qui sont placés en provinces sous la supervision du délégué du responsable de la direction des douanes et accises, et ont pour tâche d'exécuter les missions ordinaires leur confiées et de mettre fin à l'anarchie, aux désordres et aux tracasseries régnant en maître aux frontières aériennes, fluviales et terrestres. De plus, il leur est imposé de limiter au minimum absolu les effectifs engagés quotidiennement en ces lieux dont pour la direction des douanes et accises 60 agents à l'aéroport international de Ndjili, et 18 au Beach Ngobila par jour; pour l'office congolais de contrôle 30 agents à Ndjili et 18 au port Beach Ngobila; pour la direction générale d'immigration 90 agents et 65 .

Donc à ce niveau et en nous référant aux dispositions légales on peut se rendre compte sur l'effectivité des services devant œuvrer à la frontière avec la Zambie avec certitude et sur les tâches que chaque service est habilité à accomplir chaque jour qui passe.

A ce niveau déjà on constate que du côté Kasumbalesa-Congo, il y a déjà une multitude de services malgré les différents textes juridiques en la matière les réduisant à 4. Ceci crée souvent des conflits de compétence entre les services habilités à être à la douane et ceux qui n'ont pas de base juridique.

A titre exemplatif, que vient faire le parquet 2 à la douane à l'occasion des opérations de dédouanement alors que la direction générale des douanes et accises compte déjà des officiers de police judiciaire chargés de résoudre ou d'instruire les différents dossiers que le parquet 2 s'empresse à instruire. N'est-ce pas un imbroglio entre ces deux services?

Afin d'éviter que ces genres de désordre ne s'élargissent jusqu'à atteindre les relations avec les douaniers zambiens, nous suggérons une dotation des moyens financiers aux différents officiers de police judicaires de la douane affectés à la frontière avec la Zambie d'une part et des décisions politiques et juridiques fermes à l'égard de ces services qui créent la

13 KASANGA KAMUANJI. Rapport de stage effectué à l'office congolais de contrôle à Kinshasa. Février 2012. 
tracasserie, du désordre de manière à relancer les activités douanières afin de redonner à la douane son rôle de génératrice des recettes.

Et comme le droit douanier reconnaît à des autorités douanières de divers échelons de pouvoir prendre des décisions relatives à l'application de la législation douanière, notamment des autorités administratives, centrales, des autorités provinciales ainsi que celles des bureaux de douane, nous pensons qu'il y a lieu que ces différentes autorités ci-haut citées puissent se concerter en vue de proposer une marche à suivre plus crédible aux différentes frontières de la République Démocratique du Congo en général et plus particulièrement à la douane de Kasumbalesa étant donné qu'elle est parmi les plus importants postes frontaliers du pays.

5. Règles de financement du travail à la douane et moyen d'action de la douane.

En plus des moyens juridiques que constitue la législation douanière, la douane dispose de moyens humains, matériels et financiers (ressources propres et dotations) qui concourent à la réalisation des objectifs qui lui sont assignés.

En ce qui concerne les moyens financiers, la douane fonctionne comme tout autre service de l'Etat certes, étant un service générateur des recettes elle bénéficie des différentes allocations du gouvernement compte tenu de leurs performances. Mais notons que les moyens de fonctionnement mis actuellement à sa disposition sont insuffisants, notamment en ce qui concerne la surveillance des frontières nationales.

Dans ce sens, exhortant la brigade douanière à multiplier ses efforts de lutte contre la fraude, le directeur provincial du Katanga a relevé que « le Katanga a les frontières les plus poreuses de la république $\gg .{ }^{14}$

Dans le même ordre d'idées notons qu'en ce qui concerne les salaires et primes des agents des différentes douanes que regorge la République, le gouvernement les a largement améliorés comparativement aux fonds alloués au fonctionnement des douanes en République Démocratique du Congo.

\section{CONDITIONS GENERALES DE LA DOUANE EN REPUBLIQUE DEMOCRATIQUE DU CONGO EN GENERAL ET CELLE PARTAGEANT LA FRONTIERE AVEC LA ZAMBIE EN PARTICULIER.}

Ce point examinera successivement au point 1, de la situation générale de la douane en république Démocratique du Congo et particulièrement de la douane à la frontière avec la Zambie (Kasumbalesa, Mokambo, Sakania, et Kipushi.); au point 2, de la situation des postes frontaliers de la douane à la frontière avec la Zambie; au point 3, des droits de sortie des marchandises à l'exportation, au point 4 , de la base de calcul des marchandises lors de 
l'exportation des marchandises et enfin au point 5, de la base de calcul des marchandises lors de l'importation, particulièrement lors du dédouanement des véhicules.

D’entrée de jeux, il sied de spécifier que la douane à la frontière de la république Démocratique du Congo et la Zambie n'a pas de cadre juridique spécifique ni de règlement d'ordre intérieur propre ni un statut particulier. Ainsi, le code de douane et d'autres mesures d'application de ce code en vigueur s'appliquent mutatis-mutandis à cette douane. (Frontière entre la république Démocratique du Congo et la Zambie).

A chaque poste frontalier en République Démocratique du Congo doit correspondre un autre du pays voisin. Et, partout où il Ya une frontière en république Démocratique du Congo, il doit y avoir 4 services qui sont autorisés à y opérer et concernant la douane à la frontière avec la Zambie, il existe quatre principaux postes frontaliers notamment la douane de Kasumbalesa, Mokambo, Sakania, et Kipushi qui, referment presque les mêmes problèmes de fraude. Nous y reviendrons plus loin lorsque nous aborderons les problèmes spécifiques à chaque poste. En outre, il y a Kasenga et Pweto, comme succursales dépendant de la direction de Lubumbashi.

\section{Situation des postes frontaliers en république Démocratique du Congo}

Comme nous l'avons relevé ci haut, les postes frontaliers en République Démocratique du Congo renferment beaucoup d'irrégularités en ce qui concerne son rendement et la rapidité dans les opérations d'exportation et d'importation et ce, malgré l'instauration du guichet unique.

En fait, c'est dans le souci de facilitation et de simplification des procédures douanières qui a conduit à la mise en place d'un guichet unique, unité de centralisation tant des contrôles que des prospections qui ont lieu à l'occasion de l'importation et de l'exportation des marchandises. C'est la douane qui a été investie de cette mission. Les perceptions pour comptes des tiers sont réservées aux comptes des administrations et organismes concernés (art 351-352).

2. La douane à la frontière avec la Zambie.

Douane de Kasumbalesa et Sakania :

A ces deux postes frontaliers, il ya des pistes connues par les douaniers et tous les autres agents des différents services habiletés à ouvrer à la douane qui favorisent la fraude, la clandestinité notamment les pistes connues sous le nom de BILANGA à Kasumbalesa et MILEMA (expressions vulgaires expliquant les chemins de brousse pour faire passer les marchandises en esquivant le paiement des différentes taxes).

Ces pistes étaient organisées par les militaires affectés à la douane en collaboration avec certains services douaniers. Et aujourd'hui ces pistes de fraude ont demeuré et sont devenues une coutume pour certains opérateurs économiques avec motif que le dédouanement coûte très cher en République Démocratique du Congo. 
Sur ces pistes, beaucoup de marchandises, produits et autres biens de valeur passent sans être déclarés. On se rappellera ici de l'appellation «SMAGOLA » et « KATAKO» expressions zambiennes désignant les voleurs ou fraudeurs congolais de farine de maïs ou froment exploitant ces pistes.

De plus, les mouvements commencent sur ces tronçons à 19 h00 et se terminent à 5 h00 du matin c'est-à-dire une heure avant l'ouverture des services habilités à œuvrer à la douane et une heure après la fermeture de la douane.

Comme constat au sujet de ces pistes, les zambiens n'aiment jamais les exploiter. C'est d'ailleurs cela qui favorise la fraude, la clandestinité. Certains services congolais s'y placent pour arrêter les fraudeurs congolais, mais ceci n'a pas véritablement contribué à stopper ces pistes car à la place de sanction contre les fraudeurs, ces service leur arrachent les marchandises et produits et les rançonnent au cas où ces dits fraudeurs n'ont pas réussi à coopérer avec eux.

A ce constat, s'ajoute une multiplicité des services et organismes publics (18 services et organismes publics) qui opèrent à la douane nonobstant les textes légaux et règlementaires déjà pris notamment le décret $n^{\circ} 036 / 2002$ du 28 mars 2002 ainsi que l'ordre opérationnel $n^{\circ} 027 / 2008$ du 28 mars réduisant à 4 les services et organismes publics habilités à exercer aux frontières en République Démocratique du Congo.

De plus la fraude à la douane qui constitue un manque à gagner au trésor public est occasionnée par les déclarants en douane étant donné qu'ils sont les seuls qui peuvent effectuer les opérations de dédouanement à l'occasion de l'importation ou de l'exportation des marchandises. Ceux-ci fabriquent des factures en rabattant la valeur exacte de la marchandise en douane à leur profit mais, au désavantage du trésor public.

A titre exemplatif, lorsqu'un opérateur $\mathrm{X}$ veut dédouaner sa marchandise qui lui a coûté une somme de 100000 \$ comme coût d'achat plus transport, il se présente auprès d'un déclarant $\mathrm{Y}$ et ce dernier fait fabriquer une facture à l'insu de l'opérateur économique et des autorités douanières en rabattant les $100000 \$$ à $50000 \$$ de manière à payer les frais de douane équivalant à la somme de 50000 \$. Et si ce jeux passe inaperçu, c'est le trésor public qui perd au profit du déclarant. C'est pourquoi il est observé sur la ville de Lubumbashi que les opérateurs économiques puissants financièrement et politiquement ouvrent leurs propres agences en douane de manière soit à éviter ce jeu des déclarants soit encore en faisant ce jeu pour leur propre compte.

Nonobstant le caractère de fraude relevé ci- haut, la fraude aux différentes douanes en République Démocratique du Congo et à la frontière avec la Zambie est aussi l'œuvre de certaines autorités administratives et politiques qui empêchent le contrôle douanier en faveur de certains opérateurs économiques.

Même le parquet sur lequel on peut compter pour réprimer les délits économiques, entretient parfois des relations amicales avec les opérateurs économiques.

Ces interventions sont faites au profit des grands commerçants qui ont le monopole sur le marché. Ainsi, ces grands commerçants usent de leurs relations chaque fois que les 
agents de douane veulent leur demander des explications sur les écarts de conduite. ${ }^{15}$ De plus, la mauvaise politique salariale à l'égard des agents de douane, bien que ces agents aient vu leurs salaires s'améliorer largement depuis quelques années tout en demeurant insuffisant par rapport à leurs rendements, fait que ces derniers ne soient plus en mesure de bien mener leurs missions. Ils ont tendance à capitaliser le contrôle au profit du pain quotidien.

Douane à Mokambo :

Depuis l'instauration du guichet unique et la prise en compte de ce poste frontalier, ce point a rapidement été amélioré et est à ce jour l'un des postes frontaliers les plus sûrs en services malgré quelques petites irrégularités qui persistent.

A ces jours, comparativement à Kasumbalesa, Mokambo regorge une administration douanière plus fluide et plus sûre à l'occasion des opérations de dédouanement. Certes, il reste un poste peu exploité comparativement au poste frontalier de Kasumbalesa.

Par exemple, lorsque nous sommes à Mokambo et que nous voulons dédouaner un véhicule, dès lors que nous avons payé les frais demandés à la banque, on ne passe plus beaucoup de temps sans avoir quitté les installations de la douane avec le véhicule régulièrement dédouané. Nous y reviendrons plus loin avec force détaille lorsque nous aborderons la question de la base de calcul des droits. Autrement dit, comment les services douaniers calculent le montant à payer à l'occasion des opérations de dédouanement des biens.

\section{Douane de Kipushi :}

Souvent sollicitée par les congolais pour acheter les produits de base à bas prix, notamment : la pomme de terre, les chèvres, légumes, etc.

D'entrée de jeux, nous avons constaté qu'un zambien qui veut s'approvisionner au Congo, ne paie qu'une monnaie qui équivaut à 200 francs congolais aux services œuvrant à la douane zambienne. Mais pour nous congolais le droit de passage va jusqu'à 500 francs congolais pour une journée en terre zambienne que nous payons à nos services.

Et une fois en Zambie, un constat amer s'est observé. On retrouve un groupe des congolais qui se lèvent très tôt matin et achète la quasi-totalité des marchandises zambiennes de manière à les revendre plus cher aux congolais qui y arrivent après 8 heures du matin.

Certes, à ce point, la fraude organisée par des services de l'Etat congolais ne se fait pas sentir à grand échelle comme c'est le cas dans d'autres postes frontaliers au Katanga.

15 NGOYI NJIBU.L : Les exportations de la république démocratique du Congo vers l'union européenne librairie d'études juridiques africaines. volume1. p. 143. octobre 2009. 
De manière générale, les postes frontaliers en République Démocratique du Congo sont caractérisés par les détournements, désordres et ce, malgré l'amélioration des salaires des agents œuvrant à la douane.

A cet effet, en exécution des instructions strictes de son Excellence Monsieur le président de la république données aux responsables des ministères sectoriels à la réunion spéciale du 22 décembre 2010.

Ainsi, la commission politique, défense et sécurité a décidé au cours de sa réunion extraordinaire du 23 décembre 2010, notamment de l'actualisation urgente de l'ordre opérationnel $n^{\circ} 27 / 2008$ du 08 mars réduisant à 4 les services et organismes publics de l'Etat habilités à exercer aux frontières de la République Démocratique du Congo.

En fait, la préoccupation du chef de l'Etat était de procéder à la réorganisation de secteur douanier tout en partant du constat relatif à la persistance de l'anarchie, des désordres et des tracasseries dûs, entre autre, à la multiplicité des services de l'Etat aux frontières aériennes (12 services), fluviales(16 services) et terrestres (18 services) en général et particulièrement à l'aéroport international de Ndjili, au port international de Matadi, au poste frontalier de Kasumbalesa, au Beach Ngobila, au poste frontalier de Kasindi, etc. nonobstant les textes déjà pris à cet égard notamment le décret $n^{\circ}$ 036/2002 du 28 mars 2002 ainsi que l'ordre opérationnel $n^{\circ} 027 / 2008$ du 28 mars 2008 portant désignation des services et organismes publics habilités à exercer aux frontières de la République Démocratique du Congo. Et ce sont ces services et organismes publics dont les missions seront déterminées plus loin.

\section{Les droits de sortie :}

Les droits de sortie sont contenus dans l'ordonnance loi $n^{\circ} 10 / 002$ du 20 août 2010 portant code des douanes instituant un nouveau tarif des droits et taxes à l'importation. L'exportation est toute sortie des marchandises nationales ou nationalisées vers un pays étranger.

Elle peut être soumise au droit de sortie ou non selon les circonstances d'exportation ${ }^{16}$. En d'autres termes, la valeur en douane à l'exportation est celle de la marchandise au point de sortie, majorée, le cas échéant, des frais de transport jusqu'à la frontière mais non compris le montant :

a) des droits et taxes à l'exportation;

b) des taxes intérieures et charges similaires dont il a été donné décharge à l'exportateur. ${ }^{17}$

Le ministre des finances désigne les marchandises à l'égard desquelles les valeurs déclarées ne peuvent être inférieures à la valeur qu'il détermine par voie d'arrêté.

Lorsque la valeur des marchandises n'est pas fixée par arrêté, la valeur à déclarer pour application des droits de sortie est celle que les marchandises ont normalement au moment où elles quittent le territoire national. C'est-à-dire, la valeur à la frontière ou valeur au port. Cette valeur ne peut être inférieure au prix de réalisation à l'étranger représentée par la

16 NGOYI NJIBU.L :op.cit. p. 118.

17 Article 72 du code de douane. Art cité.p.38. 
moyenne des cours mondiaux connus ${ }^{18}$. Cette pratique en matière d'exportation est toujours d'application aujourd'hui.

En fait, le nouveau tarif des droits des taxes à l'exportation instituant un nouveau tarif des droits et taxes à l'exportation vise une meilleure fiscalisation des bois en grume, en différenciant les positions pour certains essences et du courant électrique et de consolider la taxation de diamant et de l'or dans le tarif.

4. Base de calcul des droits à l'exportation.

Ce sont les valeurs de base fixées par arrêté ministériel ayant les finances dans ses attributions, sur proposition de l'administration des douanes. Et, en voici les marchandises imposables et leurs taux :

- $1 \%$ pour le café;

- $1 \%$ pour l'énergie électrique;

- $1.5 \%$ pour le diamant et l'or d'exploitation artisanale : par carat (diamant) ou kg (or);

- $3 \%$ pour l'or et diamant de production industrielle;

- $5 \%$ pour les produits miniers, à l'exception du zinc, du cobalt, calcium, du cuivre, de la malachite, du germanium, de la platine, des minerais et concentrés de résines rheniferes ainsi que des minerais et leurs concentrés provenant de gites primaires obtenues par broyage, qui sont imposés à $10 \%$;

- $6 \%$ pour le bois brut.

\section{Base de calcul des droits à l'importation.}

Les droits et taxes douaniers reposent sur l'application des taux du tarif douanier (répertoire des taux des droits et taxes à percevoir édicté par voie de loi) sur la valeur des marchandises (art. 52 à 72). Des restrictions d'entrée ou de sortie du territoire ainsi que des impositions particulières peuvent être édictées sur base de l'origine géographique des marchandises. La preuve de l'origine peut être écrite.

Ainsi, par manque de clarté et de précision dans la logique des textes légaux en ce qui concerne les taux fixés à l'importation des marchandises, nous nous sommes basés sur un élément précis des marchandises qui fait couler beaucoup de salives aux paisibles citoyens à la douane avec la Zambie plus particulièrement à la douane de Kasumbalesa.

En fait, Justin MUAKA NDOMBE MAKULA dans son mémoire d'étude approfondie défendu à Kinshasa en 2005 portant sur le thème : La Douane comme instrument de protection des Droits de l'Homme, relève ce qui suit : en ce qui concerne le dédouanement à l'occasion de l'importation d'une marchandise en République Démocratique du Congo, les problèmes de manière dont l'administration est organisée ont de tout le temps occupé une place ambiguë dans les relations sociales. Il démontre comment l'histoire de la politique 
douanière a beaucoup évolué en république Démocratique du Congo jusqu'à atteindre les montants arrêtés de manière forfaitaire.

A titre exemplatif, aujourd'hui lorsque nous voulons dédouaner un véhicule de 4 places assises, le taux à payer à la douane est fixé à $2500 \$$ américain et ce malgré la valeur de la marchandise en douane.

Ceci favorise souvent un manque à gagner d'une part lorsque un particulier dédouane un véhicule qu'il a acheté à un prix très élevé ne correspondant pas à un véhicule qu'on peut dédouaner à 2500 \$ américain, d'autre part et de manière générale, la plupart des congolais n'achètent que des véhicules d'occasion de japon qui, leur valeur d'achat et de transport du japon jusqu'à Kasumbalesa ne dépassent pas les 2500 \$ américain.

Donc pour cette catégorie des personnes, le prix de $2500 \$$ américains imposé par l'autorité de manière forfaitaire est pour eux une injustice et mérite d'être révisé car pour eux ils dédouanent leur véhicules à $100 \%$ du cout d'achat et de transport.

Pour ce faire, nous suggérons une bonne politique de taxation des marchandises notamment les véhicules en se basant sur la valeur exacte en vue de permettre aux opérateurs de payer ce qu'il faut réellement au trésor public.

\section{CONCLUSION}

Comme nous venons de nous en rendre compte sur le déroulement des opérations douanières en République Démocratique du Congo en général et à la douane à la frontière avec la Zambie en particulier, les défaillances sont observées de toutes parts (agents de l'Etat opérant à la douane, opérateurs économiques, déclarants en douane, autorités administratives et politiques) qui ramènent à la discrédibilisation des services opérant à la douane suivant le décret $n^{\circ} 036 / 2002$ du 28 mars 2002 ainsi que l'ordre opérationnel $n^{\circ} 027 / 2008 \mathrm{du}$ 08 mars 2008 réduisantà 4 les services et organismes publics habilités à exercer aux frontières en République démocratique du Congo.

De ce fait, et en guise des perspectives dans le cadre de lege ferenda, la présente étude se propose toute une liste de recommandations en vue de faire cesser l'anarchie, les désordres et les tracasseries en ces lieux ou tout au plus les limiter dans le but d'assainir réellement la douane en République Démocratique du Congo en général et celle partageant la frontière avec la Zambie en particulier.

Notons que ces recommandations appellent une conjugaison d'effort de toutes les parties qui interviennent dans les opérations douanières et ces recommandations sont :

- Lutter contre les multiples formes de fraudes douanières en envisageant une politique de partenariat avec les institutions internationales spécialisées dans ce domaine de plus, en cherchant une expertise dans des Etats qui ont réussi dans le domaine de douane notamment les services de douane français;

- Améliorer les conditions de vie du citoyen congolais en général en mettant en place une bonne politique salariale qui permettrait à tout agent de nouer les deux bouts du mois qui, par conséquent mettra fin aux différentes incertitudes les plus minimales de sa vie; 
- Donner la possibilité aux opérateurs économiques d'accéder aux différentes démarches qu'effectuent les déclarants en douane à l' occasion des opérations de dédouanement dans le but de renforcer le contrôle et de permettre aux opérateurs de se familiariser aux différentes étapes de dédouanement;

- Pousser les dirigeants du pays à doter la République Démocratique du Congo d'une législation douanière ordonnée et complet qui tienne compte des réalités sociales du congolais pour que la pratique puisse accompagner les textes légaux et non adopter des lois pour améliorer l'image du pays à la face du monde;

- La volonté politique dans le chef des dirigeants congolais pour veiller à l'application effective des lois édictées en commençant par expulser des frontières les services et organismes de l'Etat non autorisés à exercer aux frontières par le décret ci- haut évoqué;

- Vulgariser le code douanier et ses mesures juridiques d'application par tous les moyens et techniques modernes existant de manière à permettre aussi aux opérateurs économiques de s'en approprier;

- Mettre sur pied une police de douane animée par un corps consciencieux, intègre, équipé en moyen de transport et de communication pour mieux maximiser sa capacité de contrôler les différents postes frontaliers que compte la République Démocratique du Congo en général;

\section{BIBLIOGRAPHIE}

\section{TEXTES LEGAUX :}

1. Décret du roi souverain du 15 décembre 1885;

2. Acte général de Berlin de 1885 ;

3. Ordres Opérationnels $n^{\circ} 027 / 2008$ du 08 mars et $n^{\circ}$ 234/2010 du 27/12/2010;

4. Décret $n^{\circ} 036 / 2002$ du 28 mars 2002 portant désignation des services et organismes publics habilités a exercé aux frontières de la république démocratique du Congo;

5. Loi $n^{\circ} 08 / 007$ du 07 juillet 2008 portant dispositions générales relatives à la transformation des entreprises publiques;

6. Loi $n^{\circ} 08 / 009$ du 07 juillet 2008 portant dispositions générales applicables aux établissements publics;

7. Loi $n^{\circ} 08 / 009$ du 07 juillet 2008 fixant les règles relatives à l'organisation et à la gestion du portefeuille de l'Etat;

8. Décret du premier ministre $n^{\circ} 09 / 11$ du 24 avril 2009 portant mesures transitoires relatives à la transformation des entreprises publiques;

9. Décret $n^{\circ} 09 / 12$ du 24 avril 2009 établissant la liste des entreprises transformées en sociétés commerciales, en établissements publics et celles dissoutes et celles à liquider; 
10. loi $n^{\circ} 78 / 002$ du 06 janvier 1978 portant organisation des entreprises publiques;

11. Ordonnance loi $n^{\circ} 10 / 002$ du 20 août 2010 portant code de douane en république démocratique du Congo.

III. OUVRAGE :

1. CLIQUENO IS Martine. Droit public économique, paris 2003;

2. LUKOMBE N, Droit congolais des sociétés, tome 1 à 4, PUC Kinshasa, 1999;

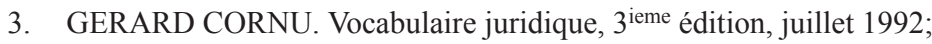

4. MAZIANI Honoré. Phénomènes de laboratoire;

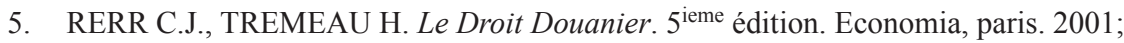

6. ESSO ABONGO, JR., la douane et les droits de douane : office des douanes et accises à l'épreuve de l'exégèse administrative. Presse de l'Institut Technique Salama. 2009;

7. JP DEMUNIEU ET ALL. Les douanes. $1^{\text {ere }}$ édition. 1959;

8. ALBERTT, J., douane et droit douanier : questions juridiques. PUF, Paris. 2013;

9. REYBROUCK D, V. Congo, une histoire, ACTES SUD, Amsterdam, 2012;

10. HOCHSCHIDA. Les fantômes du roi Léopold : un holocauste oublié, Belfond. Paris 1998 ;

IV. DIPLOME D'ETUDE APPROFONDIE :

1. Justin MUAKA NDOMBE MAKULA, La Douane comme instrument de protection des Droits de l'Homme. Kinshasa en 2005

\section{ARTICLES}

1. NGOYI NJIBU.L : Les exportations de la république démocratique du Congo vers l'union européenne librairie d'études juridiques africaines. volume1. p. 143. octobre 2009

\section{RAPPORT DE STAGE :}

1. KASANGA KAMUANJI. Rapport de stage effectué à l'office congolais de contrôle à Kinshasa. Février 2012

\section{REVUES ET AUTRES :}

1. Conférence nationale souveraines, rapport de la commission des eaux, forets, minerais et énergies. Kinshasa, palais du peuple 1992;

2. Service de nouvelles xinua, 'Zambia, république démocratique du Congo to improuve custom opératoire, 7 mai 2006; 
LA DOUANE A LA FRONTIERE DE LA REPUBLIQUE DEMOCRATIQUE DU CONGO ET LA ZAMBIE : ORGANISATION JURIDIQUE ET CONDITIONS GENERALES

3. Revue politique Afrique $n^{\circ} 129$ de mars 2013;

4. Rapport de réunion du comité de direction de l'office congolais de douane et accises. juin 2013. 